\title{
Novel Ring Compression Test Method to Determine the Stress-Strain Relations and Mechanical Properties of Metallic Materials
}

\author{
Guangzhao Han ${ }^{1}$, Lixun Cai ${ }^{*}$, Chen Bao ${ }^{1}$, Bo Liang ${ }^{2}$, Yang Lyu², Maobo Huang ${ }^{1}$ and Xiaokun Liu
}

\begin{abstract}
Although there are methods for testing the stress-strain relation and strength, which are the most fundamental and important properties of metallic materials, their application to small-volume materials and tube components is limited. In this study, based on energy density equivalence, a new dimensionless elastoplastic load-displacement model for compressed metal rings with isotropy and constitutive power law is proposed to describe the relations among the geometric dimensions, Hollomon law parameters, load, and displacement. Furthermore, a novel test method was developed to determine the elastic modulus, stress-strain relation, yield and tensile strength via ring compression test. The universality and accuracy of the method were verified within a wide range of imaginary materials using finite element analysis (FEA), and the results show that the stress-strain curves obtained by this method are consistent with those inputted in the FEA program. Additionally, a series of ring compression tests were performed for seven metallic materials. It was found that the stress-strain curves and mechanical properties predicted by the method agreed with the uniaxial tensile results. With its low material consumption, the ring compression test has the potential to be as an alternative to traditional tensile test when direct tension method is limited.
\end{abstract}

Keywords: Ring compression, Energy density equivalence, Stress-strain relation, Strength, Metallic material

\section{Introduction}

Various tubular structures are widely applied in engineering due to their high strength and stiffness to weight ratios, and high energy absorption to weight ratios, such as the fuel-cladding tubes in nuclear reactor $[1,2]$ and energy absorbers [3, 4]. Therefore, the mechanical properties such as stress $\sigma$-strain $\varepsilon$ relation and strength of their materials must be accurately evaluated and carefully considered in the design of these structures. However, it is difficult or impossible to perform tensile tests for small-volume materials and tube components. Accordingly, a new test method for non-traditional small specimens, such as small rings, is needed.

\footnotetext{
*Correspondence: lix_cai@263.net

${ }^{1}$ Applied Mechanics and Structure Safety Key Laboratory of Sichuan

Province, Southwest Jiaotong University, Chengdu 610031, China

Full list of author information is available at the end of the article
}

Ring specimens are easy sample preparation and simple operation, obtaining the mechanical properties via ring test has been the focus of research works over the last forty years. Wang et al. [5] proposed a ring hoop tension test method to acquire the hoop $\sigma-\varepsilon$ relations of nuclear fuel cladding tubes. In this test, a dog-bone specimen was machined on a ring extracted from the tube. Next, the ring was placed over two loading D-blocks that were parted by a testing machine. However, the method is not suitable for thin-walled rings, and the results are affected by the friction between the test specimen and loading D-blocks. Reddy and Reid [6-8] introduced a simple theory to obtain the yield strength via a ring compression test (RCT). The load $P$-displacement $h$ curve is obtained by applying a lateral load to the ring specimen through two rigid platens, and then the yield stress is determined according to a linear relation between the yield load and stress. However, for most metallic materials with constitutive power law, this 
method is not universally applied. With the development of simulation technology, Nemat-Alla [9] developed an experimental-numerical method to acquire the $\sigma-\varepsilon$ curves of compressed rings. In this method, an imaginary $\sigma-\varepsilon$ curve is inputted in the finite element analysis (FEA) program to predict the $P-h$ curve. Then, the test and analysis curves are compared to adjust the inputted $\sigma-\varepsilon$ relation until they agree. This method cannot be used extensively owing to the complicated iterative calculations involved. Based on the work of Nemat-Alla, Vincent et al. [10] evaluated the mechanical properties of oxidized Zircaloy-4 cladding materials and a valid accordance was presented. Chen and Cai [11-14] proposed a theoretical model for compressed rings under plane-stress conditions based on energy density equivalence. This model can predict the $\sigma-\mathcal{E}$ curves of metallic materials with constitutive power law; however, it does not give details about the elastic modulus test method, and the thickness effect of ring specimen is not considered. The longitudinal RCT has been described as "the unofficial standard" friction test method and is widely used to study the friction behavior of bulk metal forming analytically $[15,16]$, experimentally $[17,18]$ and numerically $[19,20]$. In addition, the energy absorption properties of ring structures [21-27] have been widely investigated, however, the potential of the RCT in these studies has not been highlighted due to the lack of an elastoplastic solution for compressed rings.

Hence, the main purpose of this investigation is to develop a method for three-dimensional rings that can acquire the mechanical properties of metallic materials through RCT. To achieve this, a dimensionless elastoplastic load-displacement model for compressed rings (EPLDRing) is proposed based on energy density equivalence. Solving this model using the information contained in the $P-h$ curves yielded the elastic modulus, $\sigma-\varepsilon$ relation, and strength of the tested material. Finally, the model is verified via FEA with a wide range of imaginary materials and through experiments with seven metallic materials.

\section{Theoretical Model for Ring Compression}

\subsection{Energy Density Equivalence Method}

For an unidirectionally loaded ring specimen, as shown in Figure 1, assuming that a geometric point $\mathrm{M}$ exists in the effective deformation region $\Omega$ and that the energy density of the representative volume element (RVE) at $M$ is equal to the average energy density of all RVEs in $\Omega$, we have

$$
u_{\mathrm{M}}=\int_{0}^{\varepsilon_{\mathrm{ij}-\mathrm{M}}} \sigma_{\mathrm{ij}} \mathrm{d} \varepsilon_{\mathrm{ij}}=U / V_{\mathrm{eff}}
$$

where $u_{\mathrm{M}}, \sigma_{\mathrm{ij}}$ and $\varepsilon_{\mathrm{ij}}$ are the energy density, stress tensor and strain tensor in the RVE at M, respectively; $\varepsilon_{\mathrm{ij}-\mathrm{M}}$ is

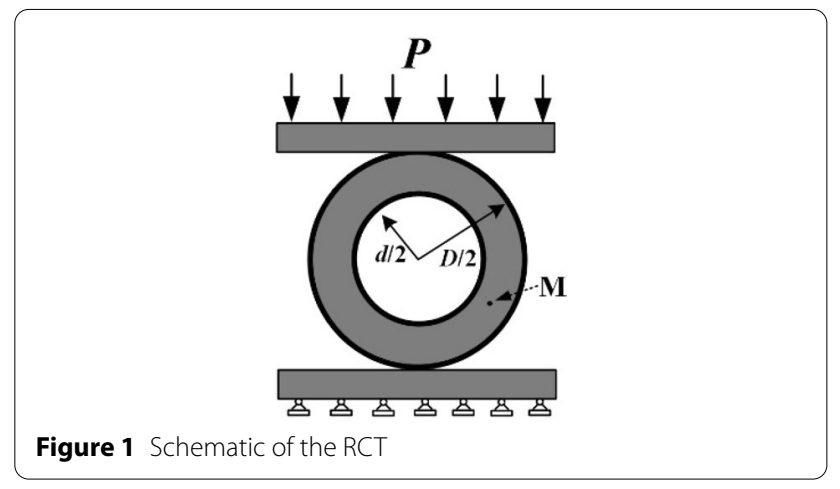

the strain tensor in the RVE at $\mathrm{M}$ in a deformation state; $V_{\text {eff }}$ is the effective volume of $\Omega ; U$ is the total deformation energy of $\Omega$, which is given by

$$
U=\iiint_{\Omega} u(x, y, z) \mathrm{d} x \mathrm{~d} y \mathrm{~d} z
$$

According to the von-Mises equivalence principle, the energy density of the RVE at M in a complex stress state is equivalent to that in a uniaxial stress state:

$$
u_{\mathrm{M}}=\int_{0}^{\varepsilon_{\mathrm{ij}}-\mathrm{M}} \sigma_{\mathrm{ij}} \mathrm{d} \varepsilon_{\mathrm{ij}}=\int_{0}^{\varepsilon_{\mathrm{eq}}-\mathrm{M}} \sigma_{\mathrm{eq}} \mathrm{d} \varepsilon_{\mathrm{eq}},
$$

where $\varepsilon_{\text {eq-M }}$ is the equivalent strain in the RVE at M in a deformation state; $\sigma_{\text {eq }}$ and $\varepsilon_{\text {eq }}$ are the equivalent stress and strain, respectively.

Combining Eqs. (1) and (3), the total deformation energy is expressed as

$$
U=V_{\text {eff }} \int_{0}^{\varepsilon_{\text {eq }-\mathrm{M}}} \sigma_{\text {eq }} \mathrm{d} \varepsilon_{\text {eq }} .
$$

\subsection{Elastoplastic Load-displacement Model}

For most isotropic, homogeneous, and power law hardening metallic materials, the equivalent $\sigma-\varepsilon$ relations are almost consistent with the Hollomon model:

$$
\sigma_{\mathrm{eq}}= \begin{cases}E \varepsilon_{\mathrm{eq}} & \varepsilon_{\mathrm{eq}} \leq \varepsilon_{\mathrm{y}}, \\ K \varepsilon_{\mathrm{eq}}^{n} & \varepsilon_{\mathrm{eq}}>\varepsilon_{\mathrm{y}},\end{cases}
$$

where $K$ is the strain-hardening coefficient $\left(K=E^{n} \sigma_{y}^{1-n}\right)$; $E$ and $n$ are the elastic modulus and strain-hardening exponent, respectively; $\sigma_{\mathrm{y}}$ and $\varepsilon_{\mathrm{y}}$ are the nominal yield stress and strain, respectively, related by $\sigma_{\mathrm{y}}=E \varepsilon_{\mathrm{y}}$.

Substituting Eq. (5) into Eq. (4), the total deformation energy $U$ is derived as follows: 


$$
U= \begin{cases}\frac{E V_{\text {eff }}}{2} \varepsilon_{\text {eq }-\mathrm{M}}^{2} & \varepsilon_{\text {eq }-\mathrm{M}} \leq \varepsilon_{\mathrm{y}}, \\ \frac{K V_{\mathrm{eff}}}{n+1}\left(\varepsilon_{\mathrm{eq}-\mathrm{M}}^{n+1}-\frac{1-n}{2} \varepsilon_{\mathrm{y}}^{n+1}\right) & \varepsilon_{\text {eq }-\mathrm{M}}>\varepsilon_{\mathrm{y}} .\end{cases}
$$

When $\varepsilon_{\text {eq-M }}$ is much larger than $\varepsilon_{\mathrm{y}}$, the value of $(1-n) \varepsilon_{y}^{n+1} / 2$ is small compared with that of $\varepsilon_{\mathrm{eq}-\mathrm{M}}^{n+1}$ and can be ignored. Next, Eq. (6) is simplified as follows:

$$
U= \begin{cases}\frac{E V_{\text {eff }}}{2} \varepsilon_{\text {eq }-\mathrm{M}}^{2} & \varepsilon_{\text {eq }-\mathrm{M}} \leq \varepsilon_{\mathrm{y}}, \\ \frac{K V_{\text {eff }}}{n+1} \varepsilon_{\text {eq }-\mathrm{M}}^{n+1} & \varepsilon_{\text {eq }-\mathrm{M}} \gg \varepsilon_{\mathrm{y}} .\end{cases}
$$

Under linear elastic and elastoplastic conditions, the following assumptions can be made:

$$
\left\{\begin{array}{l}
\frac{V_{\text {eff }} \varepsilon_{\text {eq }-\mathrm{M}}^{2}=k_{0}\left(\frac{h}{h^{*}}\right)^{2}}{} \text { Linear elastic, } \\
\left\{\begin{array}{l}
\frac{V_{\text {eff }}}{V^{*}}=k_{1}\left(\frac{h}{h^{*}}\right)^{k_{2}} \\
\varepsilon_{\text {eq }-\mathrm{M}}=k_{3}\left(\frac{h}{h^{*}}\right)^{k_{4}}
\end{array}\right. \text { Elastoplastic, }
\end{array}\right.
$$

where $V^{\prime \prime}$ is the characteristic volume, and $V^{*}=A^{*} h^{*} ; A^{*}$ and $h^{\prime \prime}$ are the characteristic area and length, respectively; $A^{*}=\beta B D\left(1-\rho^{2}\right)$, and $h^{*}=D$, where $B$ and $D$ are the thickness and diameter, respectively; $\rho$ is the diameter ratio; $\beta$ is the thickness effect coefficient; $k_{0}$ is the elastic deformation coefficient; $k_{1}$ and $k_{2}$ are the effective volume coefficient and exponent, respectively; $k_{3}$ and $k_{4}$ are the equivalent strain coefficient and exponent, respectively.

Substituting Eq. (8) into Eq. (7), $U$ is derived as follows:

$$
U= \begin{cases}\frac{E V^{*}}{2} k_{0}\left(\frac{h}{h^{*}}\right)^{2} & \text { Linear elastic, } \\ \frac{K V^{*}}{n+1} k_{1} k_{3}^{n+1}\left(\frac{h}{h^{*}}\right)^{k_{4}(n+1)+k_{2}} & \text { Elastoplastic. }\end{cases}
$$

According to the work-energy principle, $W=U$, taking the derivative of the displacement with respect to both sides of Eq. (9), the load-displacement relation is deduced as follows:

$$
P=\left\{\begin{array}{l}
E A^{*} k_{0} \frac{h}{h^{*}} \\
K A^{*} \frac{k_{1} k_{3}^{n+1}\left[k_{4}(n+1)+k_{2}\right]}{n+1}\left(\frac{h}{h^{*}}\right)^{k_{4}(n+1)+k_{2}-1}
\end{array}\right.
$$

The rearrangement of Eq. (10) is expressed as follows:

$$
\left\{\begin{array}{l}
\frac{P}{P^{*}}= \begin{cases}\xi_{\mathrm{e}} \frac{h}{h^{*}} & \text { Linear elastic, } \\
\xi_{\mathrm{ep}}\left(\frac{h}{h^{*}}\right)^{m} \quad \text { Elastoplastic, }\end{cases} \\
\left\{\begin{array}{l}
P^{*}=E A^{*} \\
\xi_{\mathrm{e}}=k_{0} \\
\xi_{\mathrm{ep}}=\frac{K}{E} \frac{k_{1} k_{3}^{n+1}(m+1)}{n+1} \\
m=k_{4}(n+1)+k_{2}-1
\end{array}\right.
\end{array}\right.
$$

where $P^{*}$ is the characteristic load; $\xi_{\mathrm{e}}$ is the dimensionless $P-h$ deformation coefficient under linear elastic deformation; $\xi_{\mathrm{ep}}$ and $m$ are the dimensionless $P-h$ deformation coefficient and exponent under elastoplastic deformation, respectively. Eq. (11) is called the elastoplastic loaddisplacement model for compressed ring (EPLD-Ring).

Moreover, according to Eq. (11), for compressed rings under ideal linear-elastic loading conditions, the loading stiffness $S_{\mathrm{L}}(=P / h)$ is expressed as follows:

$$
S_{\mathrm{L}}=\beta k_{0} B\left(1-\rho^{2}\right) E .
$$

\section{Parameter Determination}

The EPLD-Ring model is a dimensionless equation that correlates the geometric dimensions of ring $(D, \rho, B)$, Hollomon law parameters $\left(E, \sigma_{y}, n\right)$, load, and displacement. The six constants contained in the model can be directly determined via FEA for ring compression specimens with various geometric dimensions and Hollomon law parameters.

\subsection{FEA Model}

FEA for ring compression was conducted using commercial software ANSYS14.5. Considering the symmetry of the ring, a 1/4 three-dimensional FEA model was established, as shown in Figure 2. The imaginary materials inputted in the FEA program were assumed homogenous and isotropic hardening; they satisfied the von-Mises criteria, and only linear elastic deformation occurred in the dies with elastic modulus $E_{\mathrm{p}}$. Target172 and Contact173 elements were used to establish a contact pair between the lower surface of the die and the outer surface of the

Linear elastic,

Elastoplastic. 


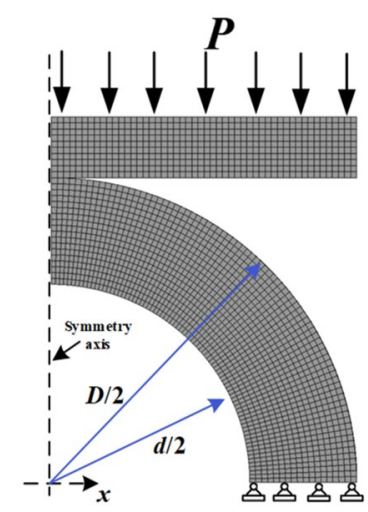

(a) Front view

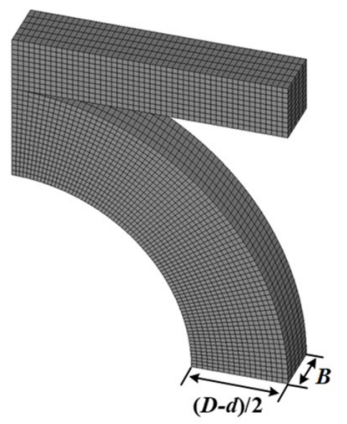

(b) Isometric view
Figure 2 FEA model for ring compression

ring, and a Solid185 element was used for the main parts of the ring and die. The cross-section of the ring and the left cross-section of the die were subjected to a symmetry constraint, and a displacement load was applied to the upper surface of the die. The influence of mesh size on the FEA model results was analyzed using various geometric rings, and the mesh size that stabilized the calculation results of the $P-h$ curves was selected.

According to the EPLD-Ring model, the $P-h$ curves can be normalized by a normalized load, $P / B D\left(1-\rho^{2}\right)$, and a dimensionless displacement, $h / D$, as shown in Figure 3. Thus, the diameter of the rings was fixed to $10 \mathrm{~mm}$ during the subsequent parameter determination steps.

\subsection{Thickness Effect Coefficient}

$\beta$ is closely related to the characteristic thickness, $B / D$, of ring specimens, and the unit thickness loading capacity

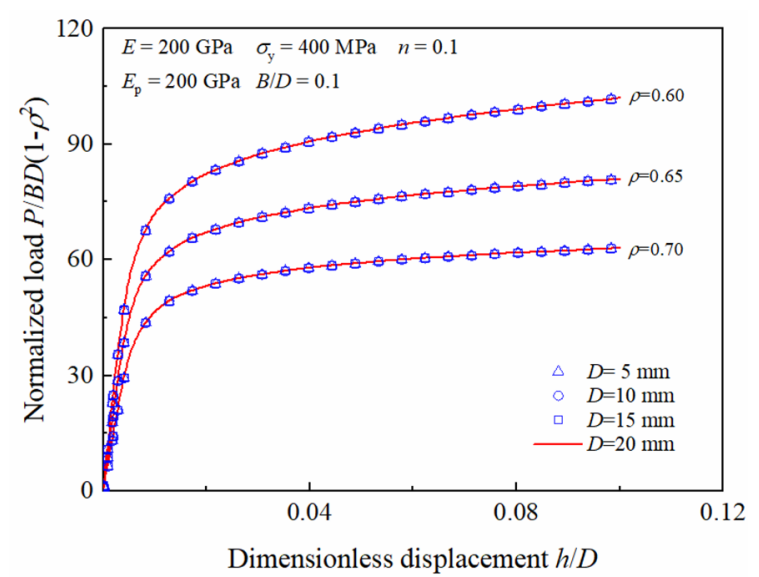

Figure 3 Normalized load-dimensionless displacement curves for various dimeters of the specimens increases with the increase in $B / D$. For various $B / D$ values, $P / B-h / D$ curves are obtained via FEA, as shown in Figure 4. Table 1 lists the FEA conditions.

In the dimensionless displacement $(h / D)$ range from 0.05 to 0.15 , fitting the $P / B-h / D$ curves of various $B / D$ values with the power law yielded the data array, $\left\{B / D, C_{\mathrm{B} / \mathrm{D}}\right\}$, where $C_{\mathrm{B} / \mathrm{D}}$ is the fitting coefficient and $C=\beta \xi_{\mathrm{ep}} E D\left(1-\rho^{2}\right)$. Using $\beta=1$ for $B / D=0.1, \beta$ values for other $B / D$ values can be obtained using $\beta \mathrm{B} / \mathrm{D}=C_{\mathrm{B} /}$ ${ }_{\mathrm{D}} / C_{0.1}$. The $\beta$ versus $B / D$ curve shown in Figure 5 and the relation between them is expressed as an arctangent equation:

$$
\beta=b_{1} \arctan \left[b_{2}\left(\frac{B}{D}\right)+b_{3}\right]+b_{4},
$$

where $b_{1}$ to $b_{4}$ are the fitting constants (see Table 2).

\subsection{Elastic Deformation Coefficient $\boldsymbol{k}_{\mathbf{0}}$}

$k_{0}$ is related to the die elastic modulus, $E_{\mathrm{p}}$, and the diameter ratio of ring specimen, and can be obtained by fitting the $P / P^{*}-h / D$ curves with a linear equation under linear elastic deformation. The $k_{0}$ versus $1-\rho$ curve was obtained via FEA with $E_{\mathrm{p}}$ fixed at $400 \mathrm{GPa}$, as shown in Figure 6(a); the relation between $k_{0}$ and $1-\rho$ can be described by the following power law equation: $k_{0}=a_{1}(1-\rho)^{a 2}$, where $a_{1}$ and $a_{2}$ are the fitting coefficient and exponent, respectively. We defined $E_{\mathrm{p}}{ }^{*}=400$ $\mathrm{GPa}$ as the characteristic elastic modulus and $k_{0}{ }^{*}=a_{1}(1-$ $\rho)^{a 2}$ as the characteristic elastic deformation coefficient. Subsequently, the $E_{\mathrm{p}} / E_{\mathrm{p}}{ }^{*}$ versus $k_{0} / k_{0}{ }^{*}$ curve shown in Figure 6(b) was obtained via FEA with $\rho$ fixed at 0.6; the relation between $E_{\mathrm{p}} / E_{\mathrm{p}}$ " and $k_{0} / k_{0}{ }^{*}$ can be described by the following power law equation: $k_{0} / k_{0}{ }^{*}=a_{3}\left(E_{\mathrm{p}} / E_{\mathrm{p}}{ }^{*}\right)^{a 4}$, where $a_{3}$ and $a_{4}$ are the fitting coefficient and exponent,

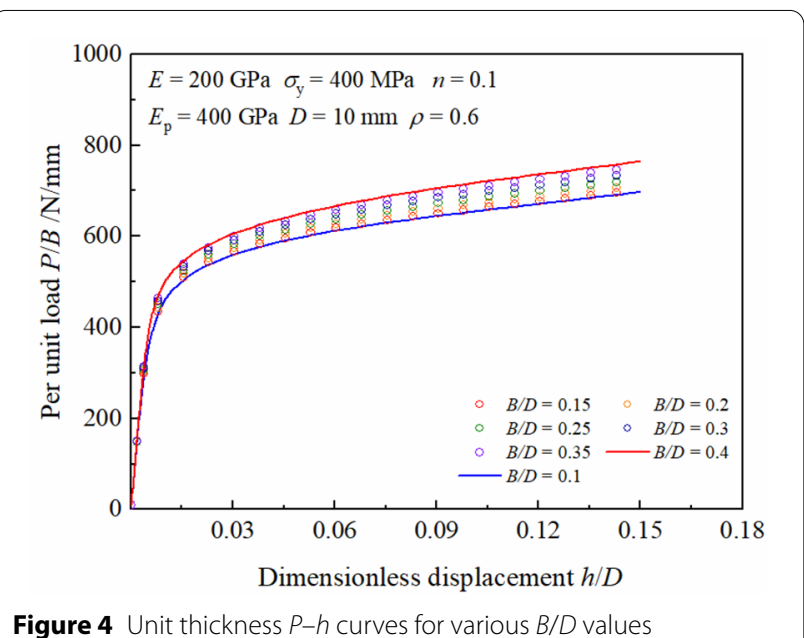


Table 1 FEA conditions for determine $\beta$

\begin{tabular}{lllllll}
\hline $\begin{array}{l}\text { Elastic modulus of } \\
\text { the die }\end{array}$ & \multicolumn{2}{l}{ Geometric size of rings } & & \multicolumn{2}{l}{ Power law parameters } \\
\cline { 2 - 5 }$E_{\mathrm{p}}(\mathrm{GPa})$ & $\boldsymbol{D}(\mathrm{mm})$ & $\boldsymbol{d}(\mathrm{mm})$ & $\boldsymbol{B}(\mathrm{mm})$ & & $\boldsymbol{E}(\mathrm{GPa})$ & $\sigma_{\mathrm{y}}(\mathrm{MPa})$ \\
\hline 400 & 10 & 6 & 1 to 4 & 200 & 400 \\
\hline
\end{tabular}

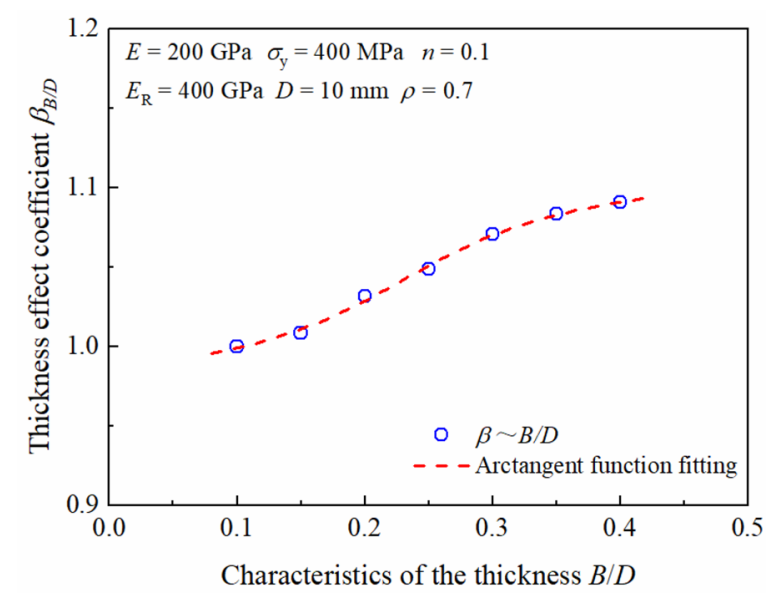

Figure $5 \beta$ versus $B / D$

respectively. Thus, the relation between $k_{0}, E_{\mathrm{p}}$, and $\rho$ is expressed as follows:

$$
k_{0}=c_{01}(1-\rho)^{c_{02}}\left(E_{\mathrm{p}} / E_{\mathrm{p}}^{*}\right)^{c_{03}}
$$

where $c_{01}, c_{02}$, and $c_{03}$ are the fitting constants, and $c_{01}=a_{1} a_{3}, c_{01}=a_{2}$, and $c_{03}=a_{4}$ (see Table 2).

\subsection{Elastoplastic Deformation Coefficients $\boldsymbol{k}_{\mathbf{1}}$ to $\boldsymbol{k}_{\mathbf{4}}$}

Because the influence of $E_{\mathrm{p}}$ on the elastoplastic $P-h$ curves is limited, $k_{1}$ to $k_{4}$ are only related to the diameter ratio, $\rho$, of ring specimens. For the ring specimens with a fixed $\rho$, these deformation constants can be determined by fitting the elastoplastic $P-h$ curves with various $n$ values. Table 3 shows the FEA conditions used to determine $k_{1}$ to $k_{4}$ for the ring specimen with a $\rho$ of 0.6 .

In the dimensionless displacement ranging from 0.05 to 0.15 , fitting the $P / P^{*}-h / D$ curves of various $n$ values with the power law yielded data arrays $\left\{n, \xi_{\text {ep }}\right\}$ and $\{n$, $m\}$. Data arrays $\left\{n+1, \xi_{\mathrm{ep}} E(n+1) / K(m+1)\right\}$, and $\{n+1$, $m+1\}$ were acquired by combining the FEA conditions and Eq. (11). By fitting the relation between $n+1$ and $\xi_{\mathrm{ep}} E(n+1) / K(m+1)$ with an exponent equation, $k_{1}$ and $k_{3}$ were determined. $k_{2}$ and $k_{4}$ were determined by fitting the relation between $n+1$ and $m+1$ with a linear equation.

Similarly, $k_{1}$ to $k_{4}$ could be directly determined for various $\rho$ values ( 0.625 to 0.7 ), as shown in Figure 7. The relations between $k_{1}$ to $k_{4}$ and $\rho$ are as follows:

$$
\left\{\begin{array}{l}
k_{1}=c_{11} \rho^{c_{12}} \\
k_{2}=c_{21} \rho^{c_{22}} \\
k_{3}=c_{31}+c_{32} \rho \\
k_{4}=c_{41}+c_{42} \rho+c_{43} \rho^{2}
\end{array}\right.
$$

where $c_{11}, c_{12}, c_{21}, c_{22}, c_{31}, c_{32}$ and $c_{41}$ to $c_{43}$ are the fitting constants (see Table 2).

In summary, the fitting constants of the EPLD-Ring model parameters are valid for rings with thickness $B$ within $[0.1 D, 0.4 D]$ and diameter ratio $\rho$ within $[0.6,0.7]$. If the geometric dimensions $(D, \rho, B)$ of a ring is measured, then $\beta$ and $k_{0}$ to $k_{4}$ can be calculated by combining Eqs. (13)-(15) and the parameters in Table 2. In fact, by re-calibrating parameters $\beta$ and $k_{0}$ to $k_{4}$, the EPLD-Ring model can be appropriate for rings whose dimensions are not within the aforementioned range.

\section{Novel Ring Compression Test Method}

\subsection{Elastic Modulus Test Method}

During the RCT, because of the interference of the initial contact nonlinearity, geometric dimension errors, and other factors, the linear elastic condition in initial loading stage of the $P-h$ curves is difficult to satisfy; therefore, the elastic modulus $E$ obtained through the initial loading stiffness $S_{\mathrm{L}}$ of the $P-h$ test curve is difficult to realize. Using the initial unloading stiffness $S_{\mathrm{u}}$ during the loading-unloading test to calculate $E$ is common in

Table 2 Fitting constants of the EPLD-Ring model parameters

\begin{tabular}{llllllll}
\hline $\boldsymbol{b}_{\mathbf{1}}$ & $\boldsymbol{b}_{\mathbf{2}}$ & $\boldsymbol{b}_{\mathbf{3}}$ & $\boldsymbol{b}_{\mathbf{4}}$ & $\boldsymbol{c}_{\mathbf{0 1}}$ & $\boldsymbol{c}_{\mathbf{0 2}}$ & $\boldsymbol{c}_{\mathbf{0 3}}$ & $\boldsymbol{c}_{\mathbf{1 1}}$ \\
\hline 0.0467 & 9.90 & -2.27 & 1.04 & 0.456 & 2.07 & 0.0630 & 0.177 \\
$c_{12}$ & $c_{21}$ & $c_{22}$ & $c_{31}$ & $c_{32}$ & $c_{41}$ & $c_{42}$ & $c_{42}$ \\
-2.07 & 0.0337 & -3.01 & 0.832 & -0.730 & 0.361 & 1.52 & -1.07 \\
\hline
\end{tabular}




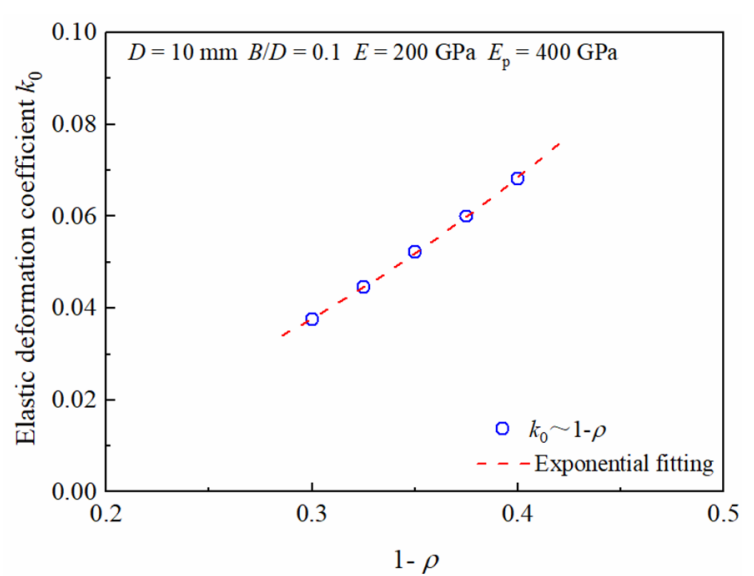

(a) $k_{0}$ versus $1-\rho$

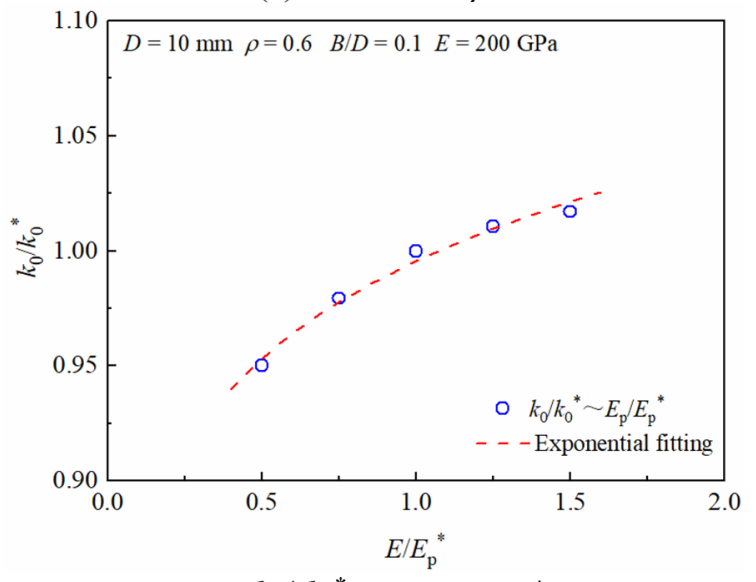

(b) $k_{0} / k_{0}{ }^{*}$ versus $E_{\mathrm{p}} / E_{\mathrm{p}}{ }^{*}$

Figure 6 Fitting relations between $\left(E_{p}, \rho\right)$ and $k_{0}$

elastoplastic indentation problem [28, 29]. Accordingly, this method was applied in our investigation.

In the loading-unloading test for ring compression, the power-law equation, $P_{\mathrm{u}}=a h_{\mathrm{u}}{ }^{t}$, was used to fit the data points of the unloading-stage $P_{\mathrm{u}}-h_{\mathrm{u}}$ curve. $S_{\mathrm{u}}$ is expressed as follows:

$$
S_{u}=\left.\frac{\mathrm{d} P_{u}}{\mathrm{~d} h_{u}}\right|_{h_{u}=h_{u \max }}=a t h_{u \max }^{t-1},
$$

where $a$ and $t$ are the fitting coefficient and exponent of the $P_{\mathrm{u}}-h_{\mathrm{u}}$ curve, respectively, and $h_{\mathrm{umax}}$ is the displacement at initial unloading point of the $P_{\mathrm{u}}-h_{\mathrm{u}}$ curve.
Results show that a proportional relation exists between $S_{\mathrm{L}}$ and $S_{\mathrm{u}}$ :

$$
S_{L}=\alpha S_{u},
$$

where $\alpha$ is the stiffness ratio, which can be determined via a multilevel loading-unloading test for rings with known $E$.

Multilevel loading-unloading tests were performed on $30 \mathrm{Cr} 2 \mathrm{Ni} 4 \mathrm{MoV}$ with $E$ is $202 \mathrm{GPa}$ and $7075 \mathrm{Al}$ with $E$ is $72 \mathrm{GPa}$. The $P-h$ curves, and $\alpha$ values for various $h / D$ values are shown in Figure 8. The results show that $\alpha$ is approximately constant in the dimensionless displacement $(h / D)$ range of 0.01 to 0.05 , and the average value $\alpha_{\mathrm{m}}$ is 0.967 . Thus, according to Eqs. (12) and (17), $E$ can be derived as follows:

$$
E=\frac{\alpha_{\mathrm{m}} S_{\mathrm{u}}}{\beta k_{0} B\left(1-\rho^{2}\right)},
$$

where $\beta$ and $k_{0}$ can be calculated with Eq. (13), Eq. (14), and the parameters in Table 2.

\subsection{Elastic Modulus Test Method}

According to Eq. (11), $\sigma_{\mathrm{y}}$ and $n$ are derived as follows:

$$
\left\{\begin{array}{l}
\sigma_{\mathrm{y}}=\left[\frac{\xi_{\mathrm{ep}} E^{1-n}(n+1)}{k_{1} k_{3}^{n+1}(m+1)}\right]^{1 /(1-n)} \\
n=\frac{(m+1)-k_{2}-k_{4}}{k_{4}}
\end{array}\right.
$$

where $k_{1}$ to $k_{4}$ can be calculated with Eq. (15) and Table 2.

For steels, $\xi_{\text {ep }}$ and $m$ are obtained using the power law equation to fit the $P-h$ curves obtained via the RCT within the $h / D$ range of $0.05-0.15$; for light alloys, the fitting range is $0.07-0.15$. Next, according to Eq. (19), the Hollomon law parameters $\sigma_{\mathrm{y}}$ and $n$ are acquired. Additionally, the strain-hardening coefficient $K$ and $\sigma-\varepsilon$ curve can be obtained according to Eq. (5). The yield strength $R_{\mathrm{p} 0.2}$ is determined by referring to the method recommended in the ISO 6892 standard, and the tensile strength [30] is calculated from the Hollomon law parameters as follows:

\begin{tabular}{|c|c|c|c|c|c|c|}
\hline \multirow{2}{*}{$\begin{array}{l}\text { Elastic modulus of } \\
\text { the die } \\
E_{\mathrm{p}}(\mathrm{GPa})\end{array}$} & \multicolumn{3}{|c|}{ Geometric dimensions } & \multicolumn{3}{|c|}{ Power-law parameters } \\
\hline & $D(\mathrm{~mm})$ & $d(\mathrm{~mm})$ & $B(\mathrm{~mm})$ & $E(\mathrm{GPa})$ & $\sigma_{y}(\mathrm{MPa})$ & $n$ \\
\hline 200 & 10 & 6 & 1 & 200 & 400 & 0.1 to 0.4 \\
\hline
\end{tabular}

$$
R_{\mathrm{m}}=K(n / \mathrm{e})^{n},
$$

Table 3 FEA conditions for determining $k_{1}$ to $k_{4}(\rho=0.6)$ 


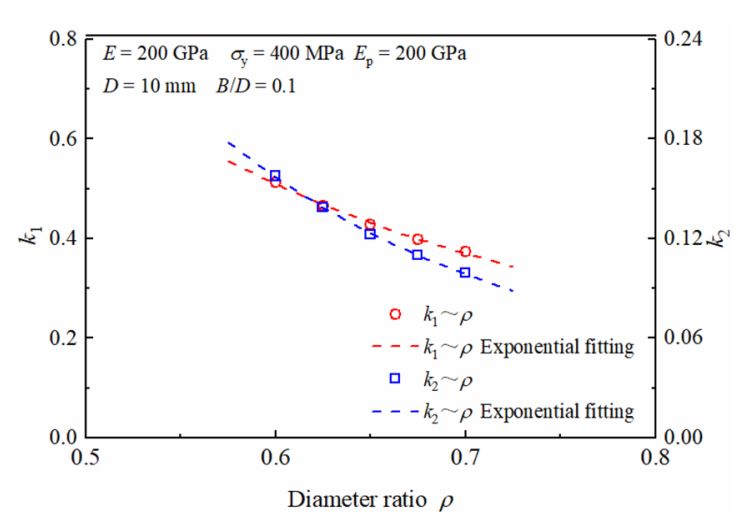

(a) $k_{1}$ and $k_{2}$ versus $\rho$

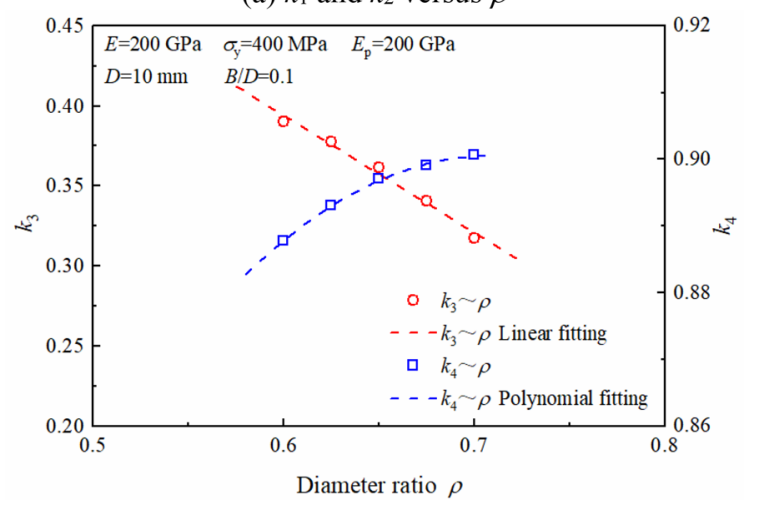

(b) $k_{3}$ and $k_{4}$ versus $\rho$

Figure 7 Fitting relations between $k_{1}$ to $k_{4}$ and $\rho$

where $\mathrm{e}$ is the natural constant, and $\mathrm{e}=2.718$.

\section{Results and Discussion}

\subsection{FEA Verification}

To verify the universality and accuracy of the novel RCT model, a wide range of imaginary materials with given constitutive relations were inputted in the FEA program for rings with various geometric dimensions. Using the method proposed in Section 4.2, the Hollomon law parameters could be acquired by fitting the $P-h$ curves obtained using FEA, and then the inputted $\sigma-\varepsilon$ curves and the predicted curves are compared and shown in Figure 9. For rings with various geometric dimensions $(D, \rho$, $B)$ and Hollomon law parameters $\left(\varepsilon_{y}, n\right)$, the goodness of fit between the predicted $\sigma-\varepsilon$ curves and the constitutive relations inputted in the FEA program were better than $99 \%$ in most case, with the lowest being $97.2 \%$ for $n=0.4$, as shown in Figure 9(c).

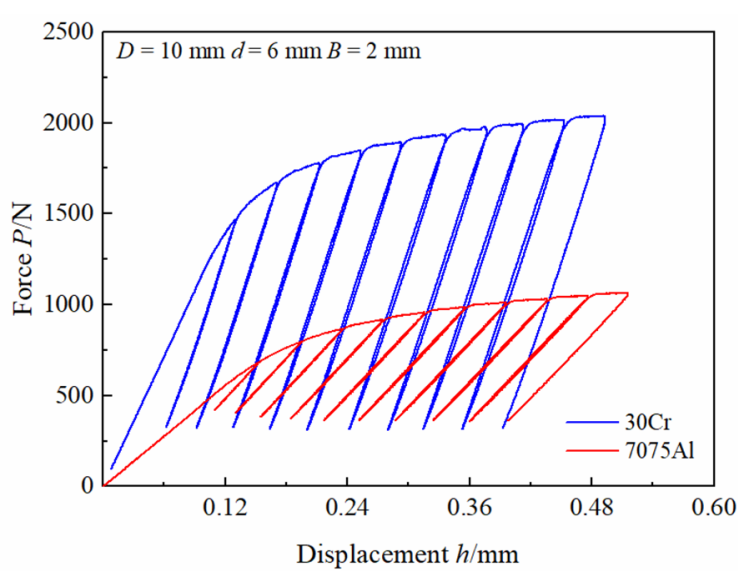

(a) Multilevel loading-unloading $P-h$ curves

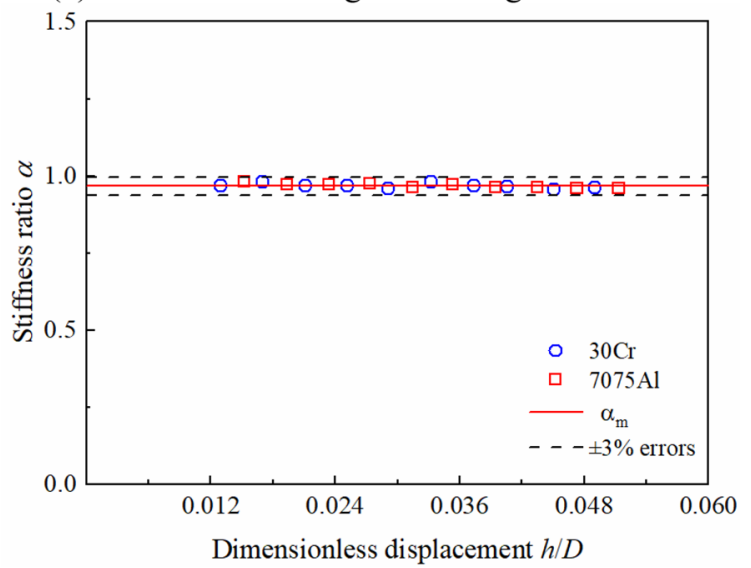

(b) $\alpha$ versus $h / D$

Figure 8 Multilevel loading-unloading tests to determine a

\subsection{Experimental Verification}

\subsubsection{Experimental Conditions}

The traditional tensile tests and RCTs were carried out on seven metallic materials, including five steels, one titanium alloy, and one aluminum alloy. Both tests were performed on an MTS 809 testing machine, an MTS 634.11F-24 extensometer was used to measure the displacement of ring specimen, as shown in Figure 10. Four specimen types were machined and the nominal dimensions of these specimens were $\{D, d, B\}$ (Unit: $\mathrm{mm}$ ): A$\{6,4,1\}, \mathrm{B}-\{8,5,1\}, \mathrm{C}-\{10,6,1\}$, and $\mathrm{D}-\{10,6,2\}$, a micrometer was used to accurately measure the dimensions of specimens before tests. The dies material was $40 \mathrm{Cr}$ with $E_{\mathrm{p}}=210 \mathrm{GPa}$. The basic mechanical properties and Hollomon law parameters of the seven metallic materials obtained by the tensile test are listed in Table 4 . 


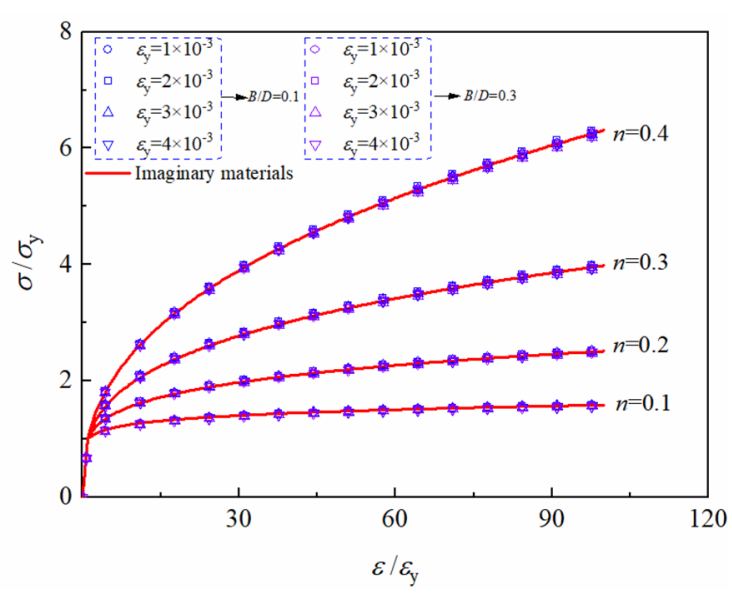

(a) $\rho=0.6$

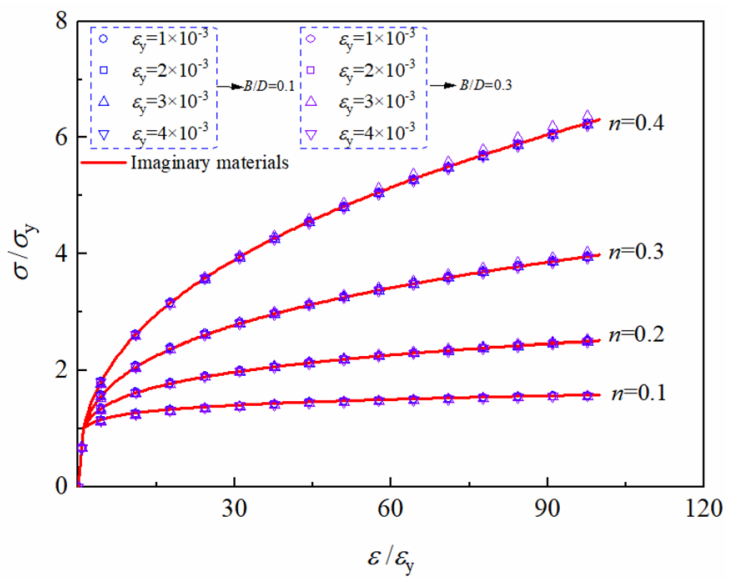

(b) $\rho=0.65$

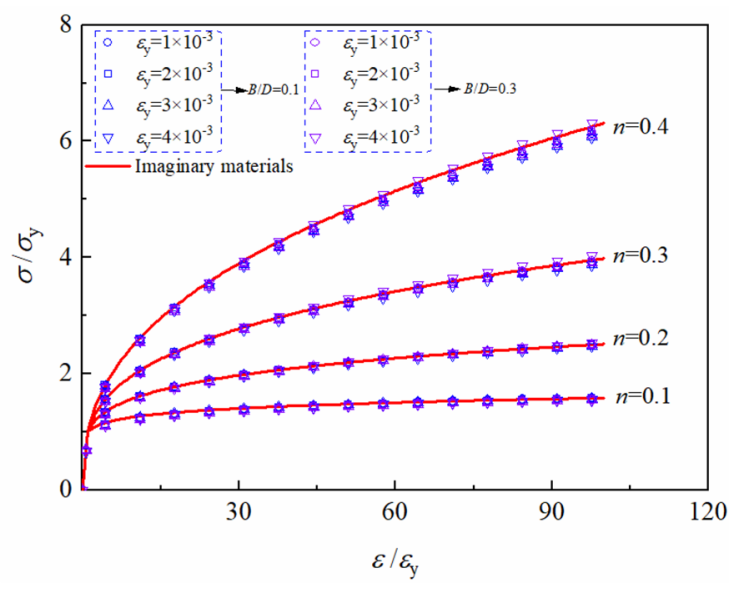

(c) $\rho=0.7$

Figure 9 Comparison between the predicted $\sigma-\varepsilon$ curves and those inputted into the FEA program

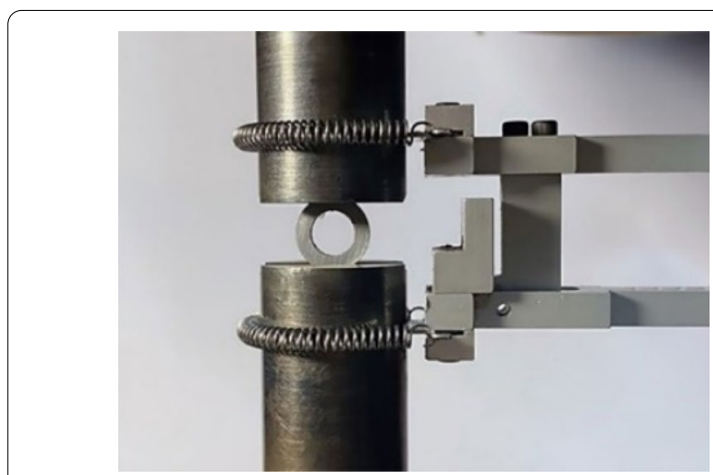

Figure 10 RCT set-up

Table 4 Basic mechanical properties and Hollomon law parameters of seven metallic materials

\begin{tabular}{lllllll}
\hline Materials & \multicolumn{2}{l}{ Basic mechanical properties } & & \multicolumn{2}{c}{$\begin{array}{l}\text { Hollomon law } \\
\text { parameters }\end{array}$} \\
\cline { 2 - 3 } \cline { 7 - 8 } & $\mathbf{E}(\mathrm{GPa})$ & $\boldsymbol{R}_{\mathrm{p} 0.2}(\mathrm{MPa})$ & $\boldsymbol{R}_{\mathrm{m}}(\mathrm{MPa})$ & & $\boldsymbol{\sigma}_{\mathbf{y}}$ & $\boldsymbol{n}$ \\
\hline Q345E & 210 & 467 & 585 & & 348 & 0.147 \\
16MnR & 210 & 300 & 515 & & 254 & 0.172 \\
A508-III & 210 & 511 & 656 & & 468 & 0.118 \\
P91 & 208 & 597 & 692 & & 562 & 0.0825 \\
30Cr & 202 & 798 & 886 & & 751 & 0.0798 \\
TC4-DT & 120 & 804 & 875 & & 800 & 0.0661 \\
7075Al & 72 & 437 & 519 & & 431 & 0.102 \\
\hline
\end{tabular}

\subsubsection{Experimental Results and Discussion}

Ring compression tests were carried out at a constant displacement rate of $0.18 \mathrm{~mm} / \mathrm{min}$. Four specimens were tested for each experimental condition, one for determining the elastic modulus $E$ using a loadingunloading test, and the remaining three for determining the Hollomon law parameters $\left(\sigma_{y}, n\right)$ using a monotonic loading test. The $P-h$ curves of both tests are shown in Figures 11 and 12, respectively.

Through the method proposed in Section 4.1, the elastic moduli $E$ of the seven materials were calculated and listed in Table 5. It can be seen that the elastic moduli predicted via ring compression test method are all close to those obtained via traditional tensile tests, and that the relative error between the two results is less than $3 \%$ for most cases, with the maximum error being 5.6\%. Additionally, multiple loading-unloading test for a single specimen can also be used to determine the elastic modulus and the average value taken as the final result. 


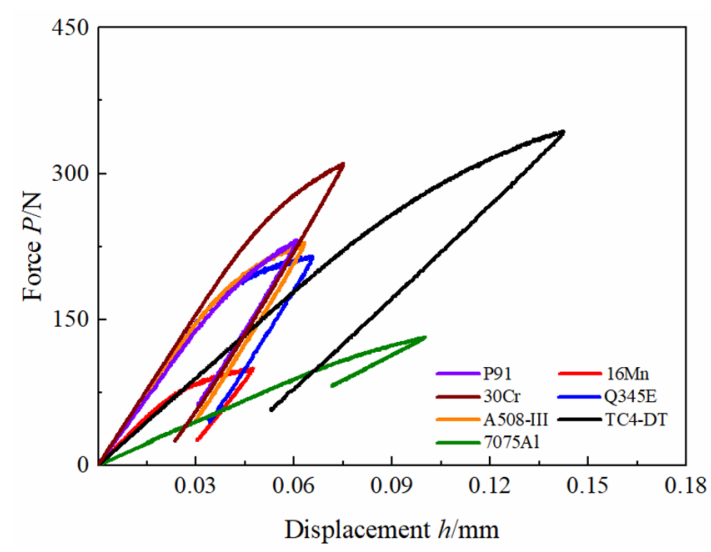

(a) Types A

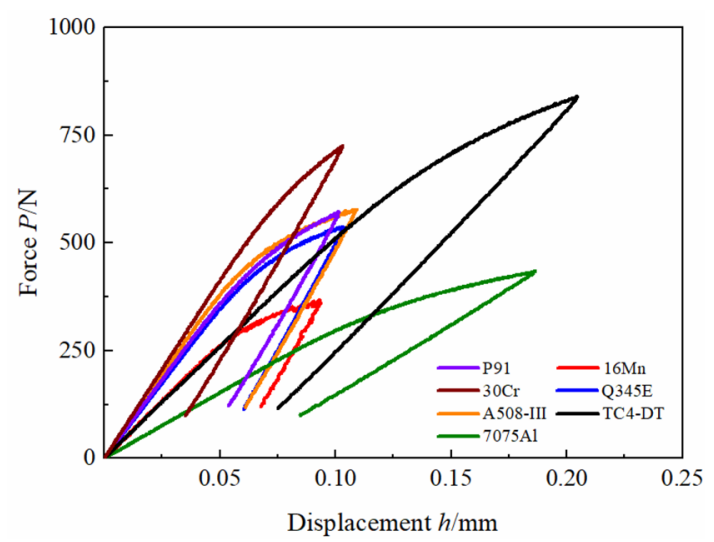

(c) Types $\mathrm{C}$

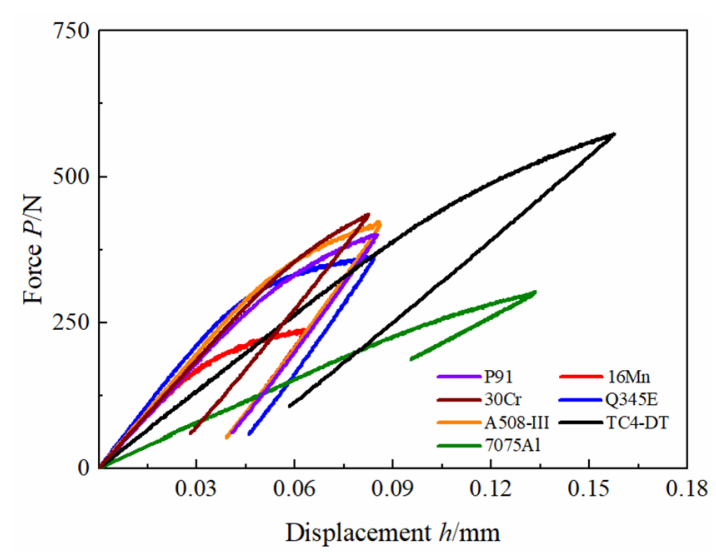

(b) Types B

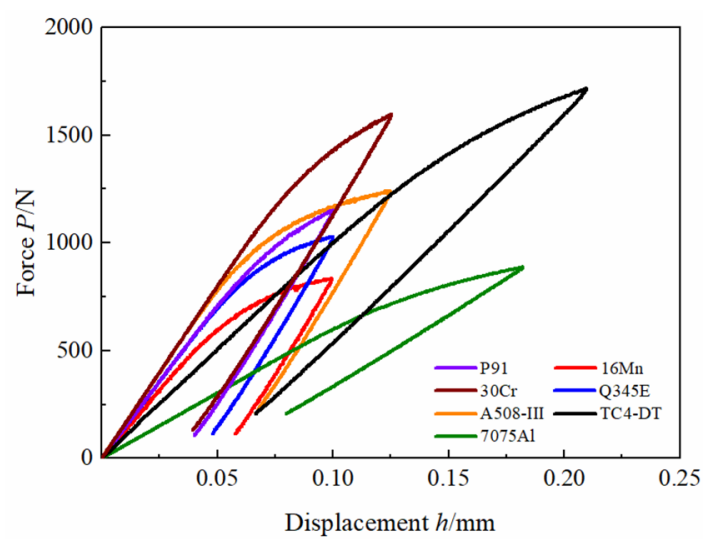

(d) Types D

Figure 11 Load-displacement curves of loading-unloading tests

Figure 12 clearly show that the $P-h$ curves of three parallel specimens with the same geometric dimensions and material are consistent; thus, according to Section 4.2, the average predicted values of the three parallel specimens is taken as the final test result, as shown in Figure 13. The predicted $\sigma-\varepsilon$ curves of the seven metallic materials are in a good agreement with the traditional tensile test results, the goodness of fit between the two curves were better than $99 \%$ in most case, with the lowest being $95.4 \%$ for $16 \mathrm{MnR}$, as shown in Figure 13(a).

Based on the method proposed in Section 4.2, the yield and tensile strengths of the seven metallic materials were obtained, as shown in Figure 14. It can be seen that the errors of yield strengths $R_{\mathrm{p} 0.2}$ obtained by RCTs and traditional tensile tests were mostly within $3 \%$ and individually within $8 \%$ for six metallic materials, except for Q345E with errors of approximately $16 \%$. The reason for this difference is that the constitutive model adopted in this study cannot be adequately describe the $\sigma-\varepsilon$ relations near the yield plateau. The tensile strengths $R_{\mathrm{m}}$ obtained by RCTs and traditional tensile tests were within $3 \%$.

In summary, for isotropic material with constitutive power law, the novel ring compression test method proposed in this study can effectively predict the elastic modulus, strengths and $\sigma-\varepsilon$ relation, and for the material whose $\sigma-\varepsilon$ relation has an obviously yield plateau, this method can used as a reference.

\section{Conclusions}

(1) In the present study, a dimensionless elastoplastic load-displacement model for three-dimensional compressed rings is proposed based on energy density equivalence. The six constants contained in the model can be determined via FEA.

(2) The compression test results for two metal rings show that the initial theoretical ring stiffness is proportional to the unloading stiffness at the unloading point of the $P-h$ curve in the $h / D$ range of 0.01 


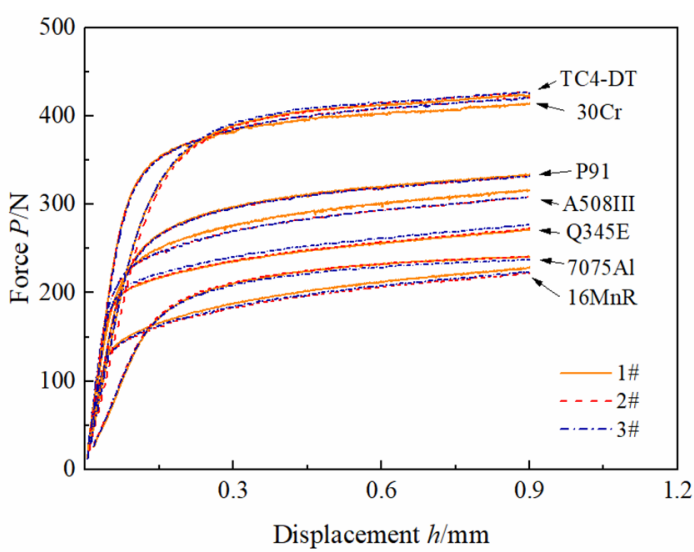

(a) Types $\mathrm{A}$

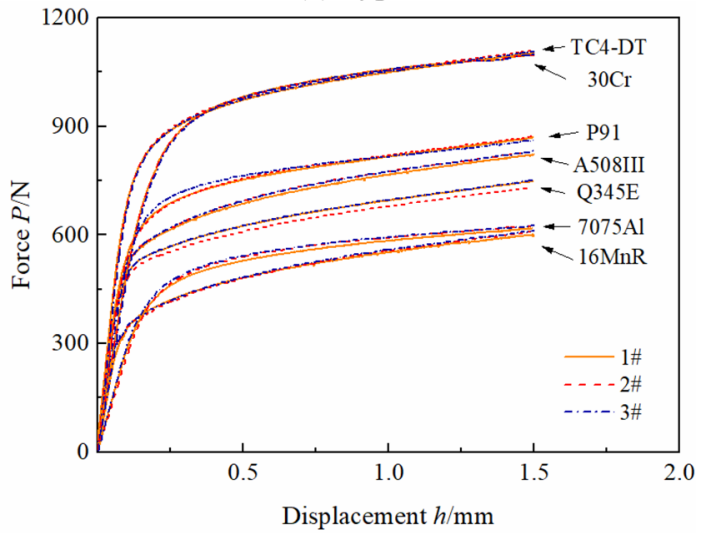

(c) Types $\mathrm{C}$

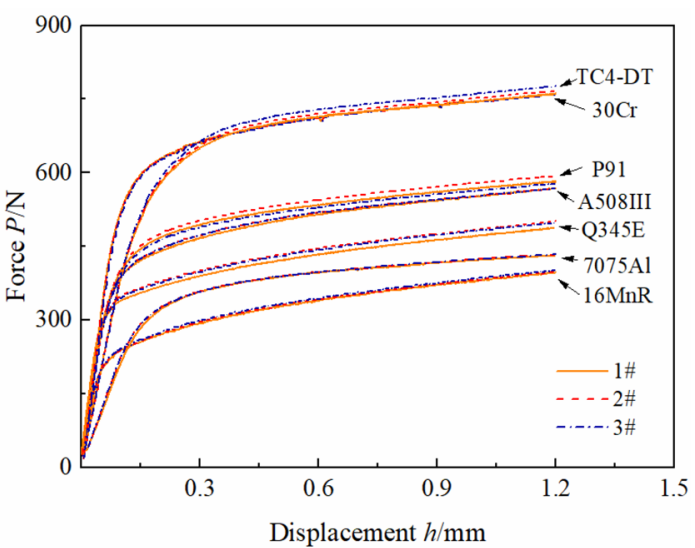

(b) Types B

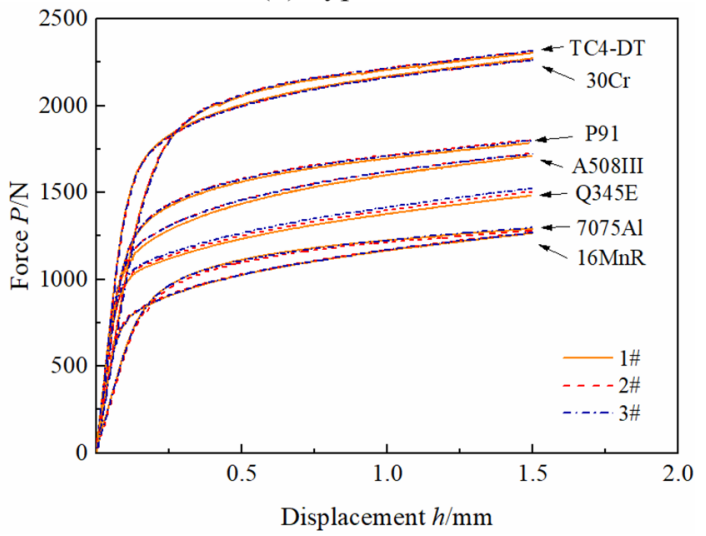

(d) Types D

Figure 12 Load-displacement curves of monotonic ring compression tests

Table 5 Comparison between the predicted elastic moduli and those obtained by traditional tensile tests

\begin{tabular}{|c|c|c|c|c|c|c|c|c|}
\hline \multirow[t]{2}{*}{ Materials } & \multicolumn{2}{|l|}{ Types A } & \multicolumn{2}{|l|}{ Types B } & \multicolumn{2}{|l|}{ Types C } & \multicolumn{2}{|l|}{ Types D } \\
\hline & $E_{\mathrm{R}}(\mathrm{GPa})$ & Errors (\%) & $E_{\mathrm{R}}(\mathrm{GPa})$ & Errors (\%) & $E_{\mathrm{R}}(\mathrm{GPa})$ & Errors (\%) & $E_{\mathrm{R}}(\mathrm{GPa})$ & Errors (\%) \\
\hline Q345E & 209 & -0.50 & 213 & 1.4 & 209 & -0.50 & 206 & -1.9 \\
\hline $16 \mathrm{MnR}$ & 207 & -1.4 & 209 & -0.5 & 214 & 1.9 & 206 & -1.9 \\
\hline A508-III & 208 & -1.0 & 202 & -3.8 & 212 & 1.0 & 207 & -1.4 \\
\hline P91 & 211 & 1.4 & 216 & 3.8 & 213 & 2.4 & 211 & 1.4 \\
\hline $30 \mathrm{Cr}$ & 198 & -2.0 & 206 & 2.0 & 205 & 1.5 & 198 & -2.0 \\
\hline TC4-DT & 119 & -0.8 & 123 & 2.5 & 124 & 3.3 & 116 & -3.3 \\
\hline $7075 \mathrm{Al}$ & 73 & 1.3 & 74 & 2.8 & 74 & 2.8 & 76 & 5.6 \\
\hline
\end{tabular}

to 0.05. Accordingly, a novel ring compression method was developed to obtain the elastic modulus, stress-strain relation, and strengths of metallic material. The method is verified via FEA with a wide range of imaginary materials and through experiments with seven metallic materials, and a valid accordance was presented.
(3) Because of their low material consumption, millimeter ring specimens have the potential to determine the mechanical properties of small-volume materials and tube components. 


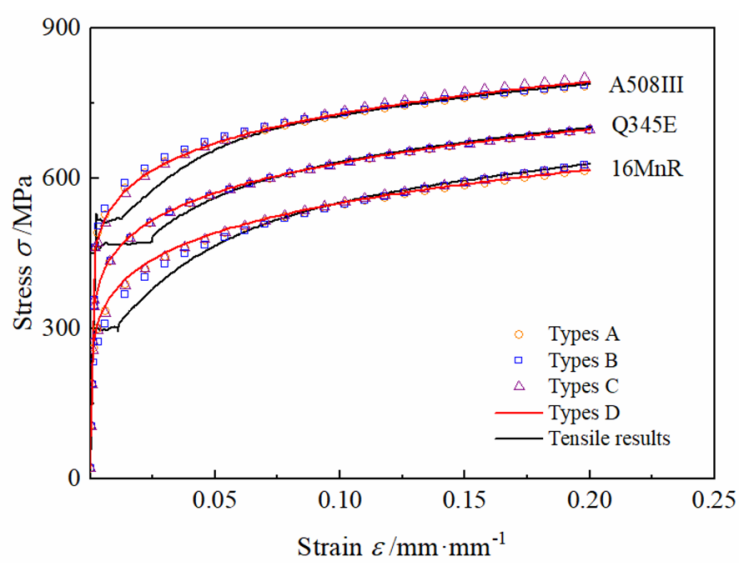

(a) Steels with a yield plateau

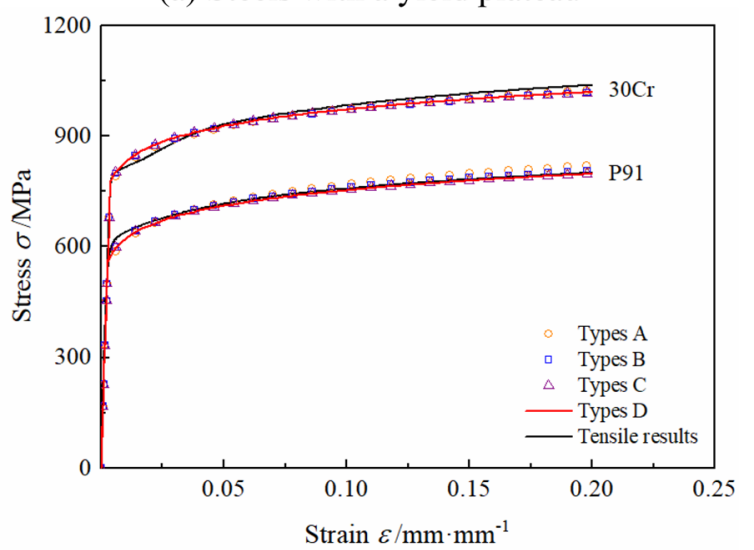

(b) $\mathrm{P} 91$ and $30 \mathrm{Cr}$

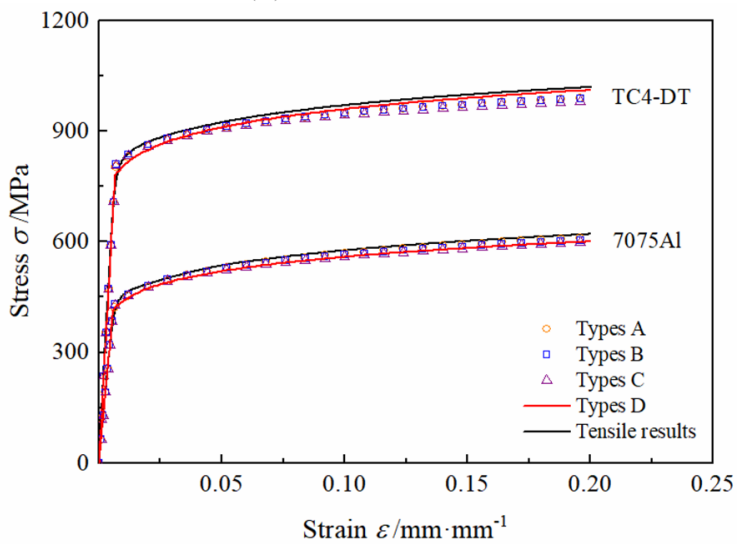

(c) TC4-DT and 7075Al

Figure 13 Comparison between the predicted $\sigma-\varepsilon$ relations $u$ and those obtained by traditional tensile tests

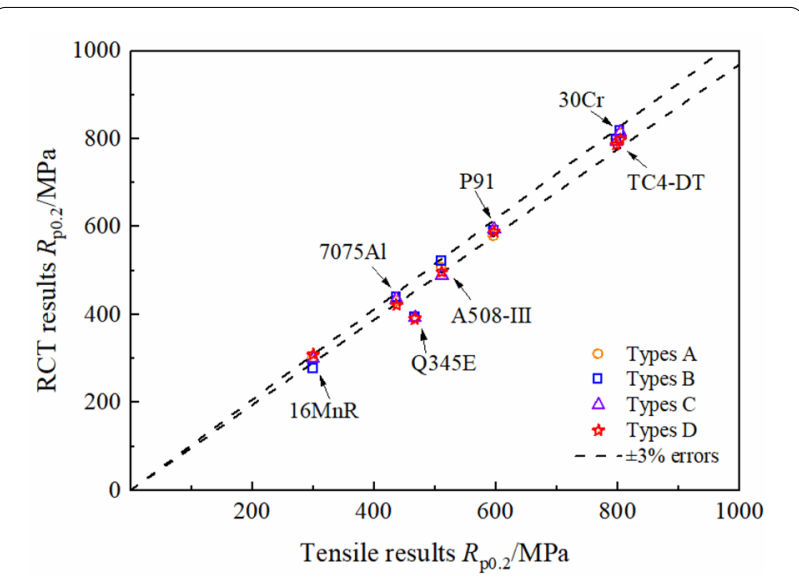

(a) $R_{\mathrm{p} 0.2 \text { results }}$

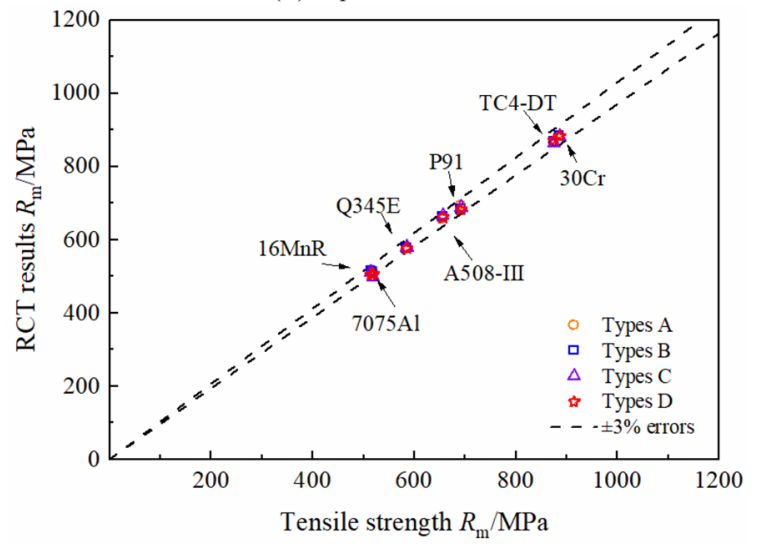

(b) $R_{\mathrm{m}}$ results

Figure 14 Comparison between the predicted yield and tensile strengths and those obtained by traditional tensile tests

\section{Acknowledgements}

Not applicable.

\section{Authors' Contributions}

GH and LC were in charge of the whole research; CB was in charge of the experiments and provided all support conditions; $\mathrm{MH}, \mathrm{BL}$ and $\mathrm{YL}$ assisted the experiments and simulations, $\mathrm{XL}$ conducted proof reading and made some revisions; All authors read and approved the final manuscript.

\section{Authors' Information}

Guangzhao Han, born in 1994, is currently a PhD candidate at Applied Mechanics and Structure Safety Key Laboratory of Sichuan Province, Southwest Jiaotong University, China.

Lixun Cai, born in 1959, is currently a professor and a PhD candidate supervisor at Applied Mechanics and Structure Safety Key Laboratory of Sichuan Province, Southwest Jiaotong University, China. His research interests include fatigue and fracture mechanics, elastoplastic mechanics theory and testing method for miniature materials.

Chen Bao, born in 1982, is currently an associate professor at Applied Mechanics and Structure Safety Key Laboratory of Sichuan Province, Southwest Jiaotong 
University, China. His research interests include structural integrity evaluation and fracture toughness testing theory.

Bo Liang, born in 1983, is currently a researcher at AVIC Touchstone Technology Testing Innovation (Dachang) Co. LTD, China.

Yang Lyu, born in 1985, is currently a research assistant at AVIC Touchstone Technology Testing Innovation (Dachang) Co. LTD, China.

Maobo Huang, born in 1998, is currently a PhD candidate at Applied Mechanics and Structure Safety Key Laboratory of Sichuan Province, Southwest Jiaotong University, China.

Xiaokun Liu, born in 1991, is currently a PhD candidate at Applied Mechanics and Structure Safety Key Laboratory of Sichuan Province, Southwest Jiaotong University, China.

\section{Funding}

Supported by National Natural Science Foundation of China (Grant Nos.

11872320 and 12072294)

\section{Competing Interests}

The authors declare no competing financial interests.

\section{Author Details}

'Applied Mechanics and Structure Safety Key Laboratory of Sichuan Province, Southwest Jiaotong University, Chengdu 610031, China. ${ }^{2}$ AVIC Touchstone Technology Testing Innovation (Dachang) Co. LTD, Langfang 065300, China.

Received: 30 December 2020 Revised: 14 September 2021 Accepted: 12 October 2021

Published online: 20 November 2021

\section{References}

[1] F J Gómez Sánchez, M M Rengel, J Ruiz-Hervias. A new procedure to calculate the constitutive equation of nuclear fuel cladding from ring compression tests. Progress in Nuclear Energy, 2017, 97: 245-251.

[2] H Jiang, J J Wang. Development of cone-wedge-ring-expansion test to evaluate the tensile hoop properties of nuclear fuel cladding. Progress in Nuclear Energy, 2018, 108: 372-380.

[3] A Airoldi, N Novak, F Sgobba, et al. Foam-filled energy absorbers with auxetic behavior for localized impacts. Materials Science and Engineering A, 2020, 788: 139500.

[4] J P Mendez, J Diaz, L Romera. Analytical and numerical crashworthiness uncertainty quantification of metallic thin-walled energy absorbers. ThinWalled Structures, 2020, 157: 1-14.

[5] RE Link, HWang, R Bouchard, et al. Ring hoop tension test (RHTT): A test for transverse tensile properties of tubular materials. Journal of Testing and Evaluation, 2002, 30(5): 382-391.

[6] TY Reddy, S R Reid. On obtaining material properties from the ring compression test. Nuclear Engineering and Design, 1979, 52(2): 257-263.

[7] SR Reid, TY Reddy. Effect of strain hardening on the lateral compression of tubes between rigid plates. International Journal of Solids and Structures, 1978, 14(3): 213-225.

[8] TY Reddy, S R Reid. Phenomena associated with the crushing of metal tubes between rigid plates. International Journal of Solids and Structures, 1980, 16(6): 545-562.

[9] M Nemat-Alla. Reproducing hoop stress-strain behavior for tubular material using lateral compression test. International Journal of Mechanical Sciences, 2003, 45(4): 605-621.

[10] V Busser, M C Baietto-Dubourg, J Desquines, et al. Mechanical response of oxidized Zircaloy-4 cladding material submitted to a ring compression test. Journal of Nuclear Materials, 2009, 384(2): 87-95.

[1 1] H Chen, LX Cai. Unified ring-compression model for determining tensile properties of tubular materials. Materials Today Communications, 2017, 13: 210-220.
[12] H Chen, LX Cai. Unified elastoplastic model based on strain energy equivalence principle. Applied Mathematical Modelling, 2017, 52: 664-671.

[13] H Chen, LX Cai. An elastoplastic energy model for predicting the deformation behaviors of various structural components. Applied Mathematical Modelling, 2019, 68: 405-421.

[14] H Chen, LX Cai. Theoretical model for predicting uniaxial stress-strain relation by dual conical indentation based on equivalent energy principle. Acta Materialia, 2016, 121: 181-189.

[15] H Kudo. Some analytical and experimental studies of axi-symmetric cold forging and extrusion-1. International Journal of Mechanical Sciences, 1961, 2(1): 102-127.

[16] J B Hawkyard, W Johnson. An analysis of the changes in geometry of a short hollow cylinder during axial compression. International Journal of Mechanical Sciences, 1967, 9(4): 163-182.

[17] D W Zhang, M C Cui, M Cao, et al. Determination of friction conditions in cold-rolling process of shaft part by using incremental ring compression test. International Journal of Advanced Manufacturing Technology, 2017, 91(9): 3823-3831.

[18] S Hirawatari, H Watari, S Nishida, et al. Evaluation of friction properties of magnesium Alloy during hot forging by ring compression test. Materials Science Forum, 2017, 889: 119-126.

[19] Y Zhu, W Zeng, M Xiong, et al. Determination of the friction factor of Ti-6Al-4V titanium alloy in hot forging by means of ring-compression test using FEM. Tribology International, 2011, 44(12): 2074-2080.

[20] A M Camacho, J Valero, M M Marín, et al. Incremental versus continuous load-lubrication procedure in the determination of friction conditions by the ring compression test. International Journal of Mechatronics and Manufacturing Systems, 2017, 10(1): 71-83.

[21] T N Tran, A Eyvazian, Q Estrada, et al. Lateral behaviors of nested tube systems under quasi-static condition. International Journal of Applied Mechanics, 2020, 12(4): 2050046.

[22] MY Fard, B Raji, D Bao, et al. An experimental study of the mechanical properties of seamless and overlapped stitched composite tubes under hydrostatic pressure, lateral compression, and impact. The Journal of Strain Analysis for Engineering Design, 2020, 55(7-8): 212-221.

[23] S A Elahi, J Rouzegar, A Niknejad, et al. Theoretical study of absorbed energy by empty and foam-filled composite tubes under lateral compression. Thin-Walled Structures, 2017, 114: 1-10.

[24] Z P Zhou, Q Fang, F Li, et al. Experimental study on cross section lateral compression energy absorption characteristics of three types of steel tubes. Applied Mechanics and Materials, 2014, 624: 228-235.

[25] A Niknejad, S A Elahi, G H Liaghat. Experimental investigation on the lateral compression in the foam-filled circular tubes. Materials and Design, 2012, 36: 24-34.

[26] A G Olabi, E Morris, M Hashmi. Metallic tube type energy absorbers: a synopsis. Thin-Walled Structures, 2007, 45(7): 706-726.

[27] A G Olabi, E Morris, M Hashmi, et al. Optimized design of nested oblong tube energy absorbers under lateral impact loading. International Journal of Mechanical Sciences, 2008, 35(1): 10-26.

[28] Z J Zhang, L X Cai, H Chen, et al. Spherical indentation method to determine stress-strain relations and tensile strength of metallic materials. Chinese Journal of Theoretical and Applied Mechanics, 2019, 51(1): 159-169. (in Chinese)

[29] X K Liu, L X Cai, H Chen, et al. Semi-analytical model for flat indentation of metal materials and its applications. Chinese Journal of Aeronautics. 2020, 33(12): 3266-3277.

[30] T S Byun, J H Hong, F F Haggag, et al., Measurement of through-thethickness variations of mechanical properties in SA508 Gr.3 pressure vessel steels using ball indentation test technique. International Journal of Pressure Vessels and Piping, 1997, 74(3): 231-238. 